\title{
COMMENT
}

\section{Materializing the future of vaccines and immunotherapy}

\section{Darrell J. Irvine}

Materials science has the potential to overcome some of the grand challenges facing the fields of immunology and immunotherapy. The floodgates are poised to open.

Biomaterials have important roles in modern health care, ranging from synthetic resorbable sutures to orthopedic implants and drug delivery devices. Beyond these established uses, many domains of medicine are likely to depend on materials to enable their full potential in the future. One such field of great importance to global health is immunology.

The immune system not only protects the body from infectious disease but also plays a part in a host of conditions of increasing incidence and morbidity, including atherosclerosis, diabetes, multiple sclerosis, rheumatoid arthritis, inflammatory bowel disease and allergies of all types. In cancer, the immune system can be both cause and cure; it contributes to chronic inflammation that promotes tumour development, but in other cancers it provides the ultimate weapon against metastatic disease. Thus, the development of ways to harness, direct or restrain immune responses has great potential for enhancing our health. Here, three grand challenges for the field of immune engineering over the next 10 years are outlined and possible solutions provided by materials are discussed. These problems, of course, only scratch the surface of those that biomaterials could help to solve within immunology and immunotherapy.

\section{Immunomodulatory cancer drugs}

The first grand challenge is the clinical translation of methods to safely and potently modulate the immune system in patients with cancer, using the full range of candidate immunomodulatory drugs at our disposal. Recent dramatic successes in the clinic, using immunoregulatory antibodies that block immunosuppressive signals in $\mathrm{T}$ cells, have heralded a new era of cancer immunotherapy ${ }^{1}$. However, this progress must be viewed with pragmatism: the majority of immunomodulatory drugs evaluated in humans until now (for example, interleukin-2 (IL-2), interferon-a, IL-12, IL-15, anti-CD28, anti-CD137 and anti-CD40), including approved drugs, are accompanied by serious toxicities. In virtually all cases, these drugs are administered systemically and toxicity arises from on-target, off-tumour broad stimulation of immune cells in the bloodstream or distal organs. Intratumoural administration is insufficient to keep soluble drugs from rapidly disseminating into the circulation. If immunooncology continues to focus on this traditional approach to drug delivery, many promising therapeutics will not reach clinical use. Further, much preclinical evidence suggests that the network of immunosuppressive pathways established by solid tumours can only be overcome by a counter-network of signals provided by combination immunotherapy - that is, the use of multiple immunomodulatory drugs in concert. It is unclear how combination immunotherapy can become a clinical reality if these drugs cannot be delivered safely.

How can we do better? There are at least two objectives to be met: immunomodulators need to be delivered to the right cells in the right tissue microenvironments so that the total dose required is minimized, and methods to minimize systemic exposure or dissemination of these potent therapeutics must be developed. Engineered materials provide numerous strategies to achieve these goals, through the use of implantable scaffolds or hydrogels that locally provide immunoregulatory cues, nanoparticles that can be injected intratumorally and remain trapped in the local tissue, or particles that are administered systemically and accumulate in tumours with systemic exposure times that are much shorter than those for free biologics such as antibodies ${ }^{2}$. A number of these conceptual approaches have been demonstrated in preclinical (mostly small animal) models, but these ideas must now be implemented in scalable, robust, manufacturable strategies to move them into clinical testing. Traditional pharmaceutical companies do not have an established infrastructure to develop these complex products, but I suspect it will take only one compelling, successful example in humans to drive this field forward.

\section{Vaccines}

A second grand challenge is in the area of vaccines. Synthetic materials have arguably made the biggest impact in immunology through their contributions to vaccine development; for example, the first widely used vaccine adjuvant was composed of aluminum salt gels, and synthetic liposomes are approved products for 
vaccines and adjuvants. Self-assembled nanoparticles comprising virus-derived and engineered proteins are the basis of licensed vaccines and promising new vaccination and immunotherapy platforms; these are being developed by both biochemists and materials scientists. Despite this plethora of advances, hurdles remain to be overcome in the area of prophylactic vaccines. A prominent example is the HIV vaccine problem: many HIV vaccinologists believe that a successful vaccine will require the activation of $\mathrm{B}$ cells that can produce broadly neutralizing antibodies (BNAbs). BNAbs recognize highly conserved regions of the native HIV envelope trimer (the 'spike' on the surface of the virus that mediates binding and entry into target cells) and can bind to the many diverse strains of HIV that circulate in the human population. Studies of $\mathrm{HIV}^{+}$ patients have shown that $\sim 20 \%$ of those infected generate these antibodies. However, numerous facts about BNAbs isolated from these patients suggest that eliciting such antibodies through vaccination will not be easy ${ }^{3}$. First, these antibodies generally only develop over a period of several years of infection. Second, many of these antibodies have unusual features and have undergone very high degrees of somatic hypermutation, the Darwinian process by which B cells intentionally mutate their antibody genes in a stochastic manner to select antibodies that bind with increasing affinity to a target protein. As a result, strategies need to be developed to promote affinity maturation and guide the induction of targeted B cell responses through synthetic vaccines. There are numerous complementary approaches to be explored: the use of nanomaterials to multivalently display antigen or to display cocktails of antigens that can immunofocus a $B$ cell response; designing nanoparticles that concentrate $\mathrm{B}$ cell- or follicular helper T cell-stimulating adjuvants in lymph nodes; and the design of biomaterials to control the kinetics of vaccine exposure in lymphoid tissues. The field has made important first steps, through the design of antigen-displaying nanomaterials that promote enhanced follicular helper $\mathrm{T}$ cell induction and durable titres of antibodies, and through the use of biodegradable nanoparticles to concentrate potent adjuvants in lymph nodes that drive germinal centre responses. Until now, levels of somatic hypermutation, direct assessments of affinity maturation and assessments of the effect of biomaterials adjuvants on antibody specificity have been limited, if examined at all. In addition, the effect of promising nanovaccine approaches on antibody effector functions beyond isotype must be examined more closely through the evaluation of changes in antibody glycosylation and other secondary structural features ${ }^{4}$.

\section{Monitoring the immune system}

A third challenge lies in the development of strategies for monitoring the immune system in humans. Clinical analysis of immune responses remains largely confined to examination of blood draws, although both extensive preclinical and clinical studies suggest that events in tissue sites or tumours are not necessarily reflected in circulating leukocyte populations or serum factors. In cancer, direct biopsies of tumours are routinely performed but are restricted to accessible tumours, and longitudinal biopsies are problematic. Thus, new methods to query immune cell function in lymphoid organs and tissues are needed. Questions of importance would be: are $\mathrm{T}$ cells present in target tissues? Is active lysis of target cells occurring? What cytokines are present? Is antigen being presented in this site? Could we detect immune activity in tissues that would predict an approaching disease flare in autoimmunity? Recently developed environment-sensitive micelles that enter tumours and release mass-encoded peptides that are excreted in the urine in response to the presence of specific enzymes in the tumour site provide a compelling example of what might be possible ${ }^{5}$. New technologies that enable us to answer these questions in patients with minimally invasive procedures would be transformative for vaccines, cancer immunotherapy and the monitoring of autoimmune disease.

\section{Outlook}

The development of biomaterials to safely and effectively modulate the immune system is an over-arching goal for a growing cadre of materials scientists, bioengineers, pharmaceutical scientists and chemists. Success in this endeavour will require partnerships between these aforementioned groups of scientists and immunologists, vaccinologists and clinicians. Important initial steps are being made to establish these collaborations. This endeavour involves challenges well known to the bioengineering community, but also new twists. Certain classic problems (for example, targeting of drugs to disease sites) may be more tractable when the focus is on modulation of host immunity against disease rather than directly targeting diseased tissue. This is because lymphocytes can be directly accessed as they recirculate through the blood. The application of advanced materials in cancer immunotherapy is particularly compelling because the potential of immunotherapy for clinical impact is now firmly established. Working together, we will surely solve the challenges described here, and many others, to shape the future of vaccines and immunotherapy.

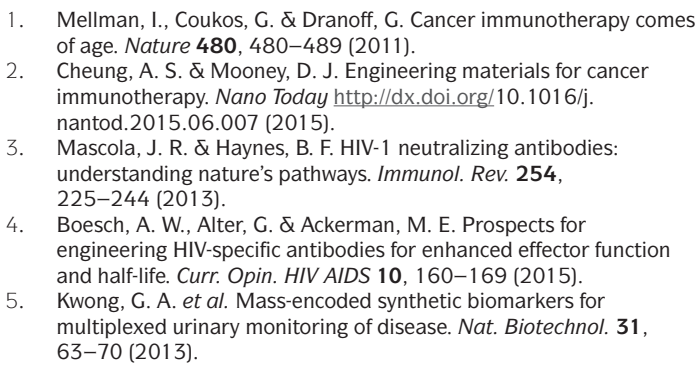

Competing interests

DJl is a co-founder of Vedantra Pharmaceuticals and Torque Biotherapeutics, and holds licensed patents in the area of vaccines and immunotherapy. 